\title{
Tipología de los consumidores de alcohol y sus factores determinantes en Bucaramanga, Colombia
}

\author{
Oscar F. Herrán ${ }^{1,2}$, María F. Ardila² \\ 1 Escuela de Nutrición y Dietética, Universidad Industrial de Santander, Bucaramanga, Colombia \\ 2 Grupo Observatorio Epidemiológico de Enfermedades Cardiovasculares, Centro de Investigaciones \\ Epidemiológicas, Universidad Industrial de Santander, Bucaramanga, Colombia
}

Introducción. En Colombia algunas consecuencias del consumo de alcohol son problemas de salud pública. Es necesario clasificar adecuadamente a los consumidores para diseñar estrategias de mitigación y control.

Objetivo. Establecer los tipos de consumidores de alcohol y sus variables asociadas.

Material y métodos. Se clasificaron 1.199 sujetos entre 18 y 60 años en tres tipos de consumo de alcohol: tipo $\mathrm{A}$, consumo deseable; tipo $\mathrm{B}$, consumo excesivo sin problemas relacionados; y tipo $\mathrm{C}$, consumo problemático o dependencia. Mediante regresión logística multinomial, se establecieron las variables asociadas a los diferentes tipos. Se discute sobre la implicación de los resultados.

Resultados. Los sujetos con expectativas positivas hacia el consumo ligadas con la expresividad y la sexualidad tienen 1,6 (IC95\%: 1,0 a 2,5) más veces probabilidad de ser tipo C que aquéllos que sin ellas; en relación con la desinhibición y los sentimientos de poder positivos, este riesgo es mayor, de 2,2 (IC95\%: 1,1 a 4,3). La edad, en una relación inversa, es factor protector para ser consumidor tipo B o C. Los hombres tienen mayor riesgo que las mujeres de tipo B o $\mathrm{C}$, al igual que los sujetos que manifestaron gusto moderado o elevado por las bebidas alcohólicas.

Conclusiones. Los resultados deben traducirse en intervenciones en la población, en grupos de riesgo como los escolares y preadolescentes con enfoque de género. El elemento por consolidar en la intervención es el de la autoestima.

Palabras clave: alcoholismo/epidemiología, consumo de bebidas alcohólicas, diagnóstico, salud pública, Colombia.

\section{Categories of alcohol consumers and the criteria for classification}

Introduction. The consequences of alcohol intake can be public health problems. A wellconstructed classification system of alcohol consumers will assist in designing strategies for mitigation and control of alcohol-induced behaviors.

Objective. A categorization of alcohol consumers was developed based on a set of consumerassociated variables.

Material and methods. A set of 1,199 subjects between 18 and 60 years old was selected and each subject classified in three categories of alcohol intake: type A, intake desirable; type $\mathrm{B}$, excessive consumption without related problems; and type $\mathrm{C}$, problematic consumption or dependence. Using multinomial logistic regression model, the decisive variables of each category were fixed

Results. Subject with positive expectations associated with consumption such as "increase in expressivity and the sexuality" have $1.6(95 \% \mathrm{Cl} ; 1.0-2.5)$ times greater probability to be placed in the $\mathrm{C}$ category that those without those expectations. For relationships associated with "inhibition and feelings of power", this risk even greater- $2.2(95 \% \mathrm{Cl} ; 1.1-4.3)$. Age is in an inverse relationship and a protective factor to be classified type $\mathrm{B}$ or $\mathrm{C}$. Men have a greater probability than women to be classes in B or $\mathrm{C}$; this probability is the same as subjects who indicate having moderate pleasure or a rise in pleasure induced by the alcoholic drinks.

Discussion. The results can be translated into programs for interventions at the population level directed to groups of higher risk, such as scholars and preteens, and with a gender focus. The 
personality element on which to focus the intervention is that of self-esteem. This is an element built from a behavioral-cognitive perspective within the context of the social and cultural learning process.

Key words: alcoholism/epidemiology, alcohol drinking, diagnosis, public health, Colombia.

Las consecuencias del consumo de alcohol han sido de interés permanente para la economía y la salud pública (1-3). Los abordajes teóricos para comprender los problemas derivados han ido desde postulados biológicos y sociales hasta culturales (4-6). A pesar del avance en la comprensión de los mecanismos que explican el consumo y las consecuencias negativas del mismo en individuos y grupos sociales, su prevención y control, son limitados (7). En Colombia durante los últimos 20 años la cantidad de alcohol consumido, el consumo problemático, la dependencia y la mortalidad y morbilidad asociadas con el consumo de alcohol, han aumentado de manera sostenida (8).

En 1999 empezó en Bucaramanga un proceso de investigación basado en aspectos cognitivos conductuales, el aprendizaje social y la cultura. Como resultado de éste, se desarrollaron y validaron instrumentos de medición para identificar las características tanto del consumo como de sus consecuencias (9-11), y más recientemente, con base en la teoría de las expectativas se establecieron las variables asociadas al consumo de alcohol (12-14).

El perfil o tipo del consumidor, del consumidor problemático y del dependiente del alcohol, ha sido objeto de estudio en la práctica clínica $(15,16)$, la epidemiológica y la salud pública $(17,18)$. La tipología obedece a un macroindicador que, de un lado, aumenta la capacidad discriminatoria y, de otro, representa integralmente un fenómeno complejo. La clasificación del consumo es un mecanismo útil en el establecimiento de relaciones causales

\footnotetext{
Correspondencia:

Oscar Fernando Herrán, Centro de Investigaciones Epidemiológicas, Facultad de Salud, Universidad Industrial de Santander, Carrera 32 № 29-31, tercer piso, oficina 304, Bucaramanga, Colombia.

Telefax: (577) 634 5781, (577) 6323215

herran@uis.edu.co, oscar.herran@gmail.com

Recibido: 23/02/09; aceptado:22/07/09
}

(19). No existen clasificaciones únicas, ellas responden a los intereses de los investigadores $\mathrm{y}$, otras veces, se derivan de aproximaciones estadísticas (19-21).

En este estudio, el interés que predomina es el de la epidemiología y la salud pública, y está íntimamente ligado a expresiones del consumo de alcohol que se consideran problemas de salud pública: el consumo excesivo, el problemático y la dependencia. Hasta ahora y para facilitar su estudio, las consecuencias del consumo de alcohol han sido consideradas como eventos independientes. Sin embargo, esto ha distorsionado la realidad y ha llevado la investigación epidemiológica a resultados contradictorios, dificultando la incorporación de éstos en las intervenciones.

El diseño de indicadores o de macroindicadores con la intención de agrupar las variables de un comportamiento que en la práctica no se pueden desligar, es una alternativa lógica para incorporar la dinámica de población en un tema tan complejo como el del consumo de alcohol. La clasificación del consumidor obedece a esta lógica y, dado el conocimiento que se tiene en Bucaramanga de las variables asociadas al consumo y del desarrollo de instrumentos de medición, se propuso integrarlo para hacer la investigación epidemiológica viable y contextualizada con la dinámica del consumidor.

Los objetivos de este estudio fueron: a) establecer los tipos de consumidores con base en la cantidad de alcohol consumida y sus consecuencias negativas, b) describir las características biológicas, sociodemográficas y dietéticas de estos tipos de consumo, o tipologías, y c) establecer las variables asociadas a estos tipos de consumo.

\section{Material y métodos}

Durante 2006, sujetos de ambos sexos con edades entre 18 y 60 años respondieron cinco 
encuestas validadas en la población adulta de Bucaramanga. Esta ciudad es el principal polo de desarrollo del nororiente colombiano, su temperatura media es de $26^{\circ} \mathrm{C}$ y cuenta con 900.000 habitantes.

\section{Tamaño de la muestra}

Se encuestaron 1.199 sujetos. El tamaño de la muestra fue definido como diez por el número de ítems del formato AEQ-III (10x120) $(22,23)$, lo que permitió confirmar la validez factorial de este cuestionario en un estudio complementario. Además, este tamaño permite, con $95 \%$ de confianza, garantizar un adecuado nivel de confiabilidad en las estimaciones de la cantidad de alcohol consumida, el consumo problemático y la dependencia del alcohol en la población.

\section{Selección de sujetos}

Los sujetos se seleccionaron mediante muestreo en múltiples etapas. Los que cumplieron los siguientes criterios, fueron elegibles:

1) edad entre 18 y 60 años,

2) residir en la ciudad dos años o más,

3) no asistir a un grupo de apoyo como alcohólicos anónimos y

4) que manifestaran su deseo de participar suministrando voluntariamente la información.

En un mapa de Bucaramanga, se eligieron al azar manzanas y, luego, casas por estrato socioeconómico; en cada vivienda se realizó un censo de elegibles y aleatoriamente se seleccionó uno por vivienda. Si esta persona se rehusaba, se elegía otra persona de la misma vivienda. Después de seleccionarlos, de informarlos sobre los objetivos, de garantizar la confidencialidad y de obtener un consentimiento escrito, se encuestaron. El proyecto y los procedimientos fueron aprobados por el Comité de Ética en Investigación de Salud de la Universidad Industrial de Santander.

\section{Fuentes de información}

Se aplicaron cinco instrumentos de medición en el siguiente orden: un cuestionario sobre aspectos biológicos, sociodemográficos y dietéticos, uno para determinar la cantidad (en gramos) del consumo semanal de alcohol durante el último año (9), la prueba de Cutting down, Annoyance by criticism, Guilty feeling, and Eye-openers (CAGE) para establecer la dependencia al alcohol (10), un cuestionario que permite establecer el consumo problemático diferente a la dependencia (CP-alcohol) (11) y, finalmente, el cuestionario AEQ-III que permite establecer las expectativas positivas hacia el consumo y que previamente fue validado en esta población $(24,25)$.

\section{Variable dependiente}

El tipo de consumo de alcohol fue la variable dependiente; este macroindicador complejo permite aproximarse a la condición "real" de la dinámica del consumidor, al integrar tres variables que son de interés para la salud pública: la cantidad de alcohol consumida (g), el consumo problemático diferente a la dependencia y la dependencia.

La tipología fue construida de la siguiente manera; primero, la cantidad de alcohol consumida se expresó en unidades internacionales de consumo (g de alcohol/10) y se generó una variable dicotómica con base en la recomendación del límite seguro de consumo semanal de alcohol (26), que para los hombres es de 28 o menos y para las mujeres es de 17 o menos unidades internacionales, respectivamente. Luego, el consumo problemático diferente a la dependencia se declaró con una respuesta positiva o más en la prueba de CP-alcohol (11), y la dependencia con tres o más respuestas positivas en la prueba de CAGE (10).

Con base en lo anterior, se establecieron tres tipos de consumo: tipo $A$, sujetos que no exceden el límite de unidades internacionales de consumo semanal de alcohol y son negativos para CPalcohol y CAGE; tipo B, quienes exceden el límite de unidades internacionales de consumo semanal de alcohol y son negativos para CPalcohol y CAGE; tipo C, quienes son positivos para CP-alcohol y CAGE, sin importar si exceden o no el límite de unidades internacionales de consumo semanal de alcohol (figura 1). 


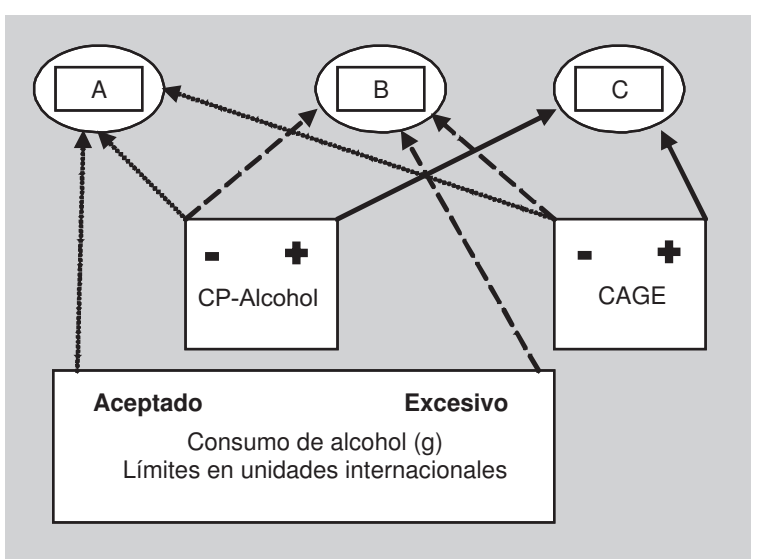

Figura 1. Configuración de la tipología del consumidor de alcohol.

\section{Análisis estadístico}

El análisis se realizó en etapas. Primero, se describieron las variables con el fin de caracterizar la población. También se describieron las características de los sujetos de acuerdo con su tipo de consumo, utilizando las pruebas de $T$ de Student, $\chi^{2}$ y ANOVA, y reportando intervalos de confianza del 95\% (IC95\%). Los estratos socioeconómicos 1 y 2 , los más bajos en la escala de ingreso y desarrollo, se agruparon en el nivel socioeconómico 1 , los 3 y 4 , en el nivel 2, y los estratos 5 y 6 , en el nivel 3. La segunda etapa tuvo como objetivo establecer la relación entre la cantidad de alcohol consumida (g), el puntaje alcanzado en las expectativas y la clasificación de las mismas con el tipo de consumo y según el sexo. Finalmente, para establecer el nivel de asociación de las variables y el tipo de consumo, se calcularon razones de disparidad para las prevalencias (OR) con sus intervalos de confianza del 95\%.

El tipo de consumo A se consideró como el de base para el modelo. Las variables fueron candidatas para entrar al modelo, si en una relación bivariada con el tipo de consumo alcanzaban un valor de $p$ igual o menor de 0,20 (27). Para el modelo final, se estableció la bondad de su ajuste mediante el cálculo de los estadísticos ${ }^{\wedge} C$ de Hosmer-Lemeshow y $\chi^{2}$ de Pearson, y el cálculo de la probabilidad (p) de que los datos predichos representen adecuadamente a los observados $(28,29)$. El modelo final se evaluó en diferentes grupos de edad, para valorar su consistencia y capacidad de explicación en ellos.

La información se sometió a doble digitación y se validó en Epilnfo v 6.04d (30). Dado el diseño de la muestra, la estimación de los errores se realizó utilizando el ajuste de la varianza de Taylor $(31,32)$. Las variables y los cálculos estadísticos se procesaron con STATA/SE versión 10.1 (33).

\section{Resultados}

\section{Población estudiada}

Como se anticipa en los estudios de población, la tasa de participación fue mayor en el nivel socioeconómico uno y disminuyó en los niveles dos y tres: $72,4 \%, 63,7 \%$ y $55,6 \%$, respectivamente. No se demostró que las personas que rechazaron participar fueran diferentes a las encuestadas; el temor a abrirle la puerta al encuestador por la inseguridad es la principal causa de rechazo.

\section{Características de la población estudiada}

Los errores estándar utilizados para calcular los IC se ajustaron por el tipo de muestreo y son equivalentes a los obtenidos en un muestreo aleatorio simple. El $49 \%$ de los entrevistados fueron hombres. La edad media de la muestra fue de 35,4 años (IC95\%: 34,4 a 36,5). El 6,4\% no terminó la educación básica primaria.

La edad media de inicio en el consumo de alcohol en los hombres fue de 15,7 años (IC95\%, 15,4 a 16,0) y en las mujeres de 18,3 años (IC95\%, 17,9 a 18,8) $(p<0,001)$. La edad media a la primera embriaguez en los hombres fue de 16,7 años (IC95\%, 16,4 a 17,0) y en las mujeres de 20,9 años (IC95\%, 20,2 a 21,6), un año después del inicio en los hombres y dos en las mujeres $(p<0,001)$. El $17,9 \%$ de los hombres y el $25,2 \%$ de las mujeres modificaron su alimentación durante el último año por sugerencia de un médico $(p=0,002)$. El $21 \%$ de los hombres y el $32 \%$ de las mujeres manifestaron hacer algún tipo de dieta en el último año $(p<0,001)$. El $42,7 \%$ presenta sobrepeso u obesidad. 
Cuadro 1. Características de la población estudiada.

\begin{tabular}{|c|c|c|c|}
\hline Característica & $\begin{array}{c}\text { Total } \\
(1.199)\end{array}$ & $\begin{array}{c}\text { Hombre } \\
\text { (588) }\end{array}$ & $\begin{array}{l}\text { Mujer } \\
(611)\end{array}$ \\
\hline Edad (años) & $35,9(35,2 \text { a } 36,6)^{*}$ & $35,4(34,4$ a 36,5$)$ & $36,3(35,4$ a 37,3$)$ \\
\hline Escolaridad (años) & $11,5(11,3$ a 11,8$)$ & $12,0(11,6$ a 12,3$)$ & $11,1(10,8$ a 11,5$)$ \\
\hline IMC (kg/m2) & $24,7(24,4$ a 25,0$)$ & $24,9(24,4$ a 25,4$)$ & $24,5(24,1$ a 24,9$)$ \\
\hline \multicolumn{4}{|l|}{ Edad (años) } \\
\hline Hasta 24 & $316\{26,3\} \dagger$ & $169\{28,7\}$ & $147\{24,1\}$ \\
\hline 25 a 39 & $423\{35,3\}$ & $201\{34,2\}$ & $222\{36,3\}$ \\
\hline 40 y más & $460\{38,4\}$ & $218\{37,1\}$ & $242\{39,6\}$ \\
\hline \multicolumn{4}{|l|}{ Escolaridad $\ddagger$} \\
\hline Menos de 5 & $76\{6,4\}$ & $36\{6,1\}$ & $40\{6,6\}$ \\
\hline 5 a 10 & $293\{24,4\}$ & $123\{20,9\}$ & $170\{27,8\}$ \\
\hline 11 o más & $830\{69,2\}$ & $429\{73,0\}$ & $410\{65,6\}$ \\
\hline \multicolumn{4}{|l|}{ IMC (kg/m2) ‡ } \\
\hline Menor de 25 & $687\{57,3\}$ & $317\{53,9\}$ & $370\{60,5\}$ \\
\hline 25 a 29 & $385\{32,1\}$ & $219\{37,2\}$ & $166\{27,2\}$ \\
\hline 30 o más & $127\{10,6\}$ & $52\{8,9\}$ & $75\{12,3\}$ \\
\hline \multicolumn{4}{|l|}{ Nivel socioeconómico | } \\
\hline Bajo & $399\{33,3\}$ & $188\{32,0\}$ & $211\{34,5\}$ \\
\hline Medio & $407\{33,9\}$ & $201\{34,2\}$ & $206\{33,7\}$ \\
\hline Alto & $393\{32,2\}$ & $199\{33,8\}$ & $194\{31,8\}$ \\
\hline \multicolumn{4}{|c|}{ Consumo de alcohol en el último año $¥$} \\
\hline Sí & $815\{68,0\}$ & $487\{82,8\}$ & $328\{53,7\}$ \\
\hline Tipo de consumo & ${ }^{* *} \ddagger$ & & \\
\hline A & $506\{42,2\}$ & $157\{26,7\}$ & $349\{57,1\}$ \\
\hline B & $475\{39,6\}$ & $270\{45,9\}$ & $205\{33,6\}$ \\
\hline C & $218\{18,2\}$ & $161\{27,4\}$ & $57\{9,3\}$ \\
\hline \multicolumn{4}{|l|}{ Consumo problemático ¥ } \\
\hline Sí & $167\{13,9\}$ & $123\{20,9\}$ & $44\{7,2\}$ \\
\hline \multicolumn{4}{|c|}{ Dependencia (según CAGE) ‡ } \\
\hline Sí & $117\{9,8\}$ & $91\{15,5\}$ & $26\{4,3\}$ \\
\hline \multicolumn{4}{|c|}{ Incremento de la expresividad y la sexualidad } \\
\hline - Puntaje alcanzado & $0,36(0,34$ a 0,39$)$ & $0,37(0,33$ a 0,41$)$ & $0,36(0,32$ a 0,39$)$ \\
\hline - Expectativa positiva & $435\{36,3\}$ & $218\{37,1\}$ & $217\{35,5\}$ \\
\hline \multicolumn{4}{|c|}{ Disminución de la tensión física } \\
\hline - Puntaje alcanzado ‡ & $0,49(0,46$ a 0,51$)$ & $0,52(0,48$ a 0,56$)$ & $0,46(0,42$ a 0,50$)$ \\
\hline - Expectativa positiva $\ddagger$ & $583\{48,6\}$ & $304\{51,7\}$ & $279\{45,7\}$ \\
\hline \multicolumn{4}{|c|}{ Desinhibición y sentimientos de poder } \\
\hline - Puntaje alcanzado ‡ & $0,84(0,81$ a 0,86$)$ & $0,87(0,84$ a 0,89$)$ & $0,80(0,77$ a 0,84$)$ \\
\hline - Expectativa positiva $\ddagger$ & $1.002\{83,6\}$ & $510\{86,7\}$ & $492\{80,5\}$ \\
\hline \multicolumn{4}{|c|}{ Disminución de la tensión psicológica } \\
\hline - Puntaje alcanzado ‡ & $0,56(0,54$ a 0,59$)$ & $0,61(0,57$ a 0,65$)$ & $0,52(0,48$ a 0,56$)$ \\
\hline - Expectativa positiva $\ddagger$ & $678\{56,6\}$ & $358\{60,9\}$ & $320\{52,4\}$ \\
\hline
\end{tabular}

* Promedio e intervalo de confianza del 95\%. † Número $\{\%\}$. | Con base en la oficina de planeación municipal, el nivel bajo se corresponde con los estratos socioeconómicos 1 y 2 , el medio con los estratos 3 y 4 y el alto con los estratos 5 y 6 . ** Tipo de consumo; A: No excede el límite de unidades internacionales de consumo semanal de alcohol y es negativo para CPA y CAGE. B: Excede el límite de unidades internacionales de consumo semanal de alcohol y es negativo para CPA y CAGE. C: Es positivo para CPA, CAGE o ambos, sin importar si excede o no el límite de unidades internacionales de consumo semanal de alcohol. $\ddagger p<0,05$.

Los hombres presentan 2,9 veces más riesgo de consumo problemático diferente a la dependencia y 3,6 veces más riesgo de alcoholismo, según CAGE, que las mujeres. El $42,2 \%$ de los encuestados fueron de tipo $\mathrm{A}$ de consumo de alcohol, el $39,6 \%$ de tipo B y el $18,2 \%$ de tipo C.

Entre los consumidores, el promedio de consumo semanal de alcohol en unidades internacionales es de 9,9 (IC95\%, 8,8 a 10,9), de 12,3 (IC95\%, 
10,7 a 13,9) en los hombres y de 6,2 (IC 95\%, $5,1$ a 7,3 ) en la mujeres ( $p<0,001)$. El $70,1 \%$ de los hombres y el $40,9 \%$ de las mujeres exceden el límite recomendado como seguro de ingesta semanal de alcohol en unidades internacionales. Otras características de la población estudiada se presentan en el cuadro 1.

\section{Características de los sujetos según su tipo su consumo}

Tipo A: el $57,1 \%$ son mujeres. La edad media fue de 39,7 años (IC95\%, 38,6 a 40,8); la escolaridad media fue de 11,0 años (IC95\%, 10,6 a 11,4); el $36 \%$ pertenece al nivel socioeconómico uno, el $34 \%$ al dos y el $30 \%$ al tres. El $24,9 \%$ de los sujetos ha cambiado su alimentación durante el último año por recomendación médica y el $29,8 \%$ ha hecho algún tipo de dieta.

El 37,3\% reconoció consumir alcohol durante el último año, el $54,7 \%$ tiene un índice de masa corporal (IMC) que puede considerarse como normal y el $13,0 \%$ son obesos. El $98,4 \%$ reconoció una frecuencia de consumo mensual y el $1 \%$ de una semanal. El $84,2 \%$ manifestó poco gusto por el alcohol y el $14,4 \%$ gusto moderado. El puntaje medio acumulado en las cuatro expectativas establecidas fue de 9,8 (IC95\%, 9,3 a 10,3). La edad media de inicio en el consumo de alcohol fue a los 17,8 años (IC95\%, 17,4 a $18,2)$ y la de la primera embriaguez los 19,8 años (IC95\%, 19,1 a 20,5); la diferencia media fue de 2,8 años.

Tipo B: el 33,6\% son mujeres. La edad media fue de 34,6 años (IC95\%, 33,4 a 35,7); la escolaridad media fue de 12,3 años (IC95\%, 11,9 a 12,7); el $27 \%$ pertenece al nivel socioeconómico uno, el $35 \%$ al dos y el $38 \%$ al tres. El $19,2 \%$ de los sujetos ha cambiado durante el último año su alimentación por recomendación médica y el $25 \%$ ha hecho algún tipo de dieta.

El $88 \%$ reconoció consumir alcohol durante el último año, el $58,9 \%$ tiene un IMC que puede considerarse como normal y el $9,5 \%$ son obesos. El 74,3\% reconoció una frecuencia de consumo mensual y el $10,9 \%$ una semanal. El $48 \%$ manifestó poco gusto por el alcohol y el $47,4 \%$ gusto moderado. El puntaje acumulado en las cuatro expectativas establecidas es en promedio de 9,9 (IC95\%, 9,5 a 10,4). La edad media de inicio en el consumo de alcohol fue de 16,9 años (IC95\%, 16,5 a 17,3) y la de la primera embriaguez de 18,3 años (IC95\%, 17,8 a 18,9); la diferencia media fue de 1,9 años.

Tipo C: el 9,3\% son mujeres. La edad media fue de 30,2 años (IC95\%, 28,7 a 31,6); la escolaridad media fue de 11,2 años (IC95\%, 10,6 a 11,8); el $40,4 \%$ pertenece al nivel socioeconómico uno, el $31,2 \%$ al dos y el $28,4 \%$ al tres. El $19,3 \%$ de los sujetos ha cambiado durante el último año su alimentación por recomendación médica y el $21,6 \%$ ha hecho algún tipo de dieta.

El 95,4\% reconoció consumir alcohol durante el último año, el 59,6\% tiene un IMC que puede considerarse como normal y el $7,3 \%$ son obesos. El 39\% reconoció una frecuencia de consumo mensual y el $39,4 \%$ una semanal. El $27,1 \%$ manifestó poco gusto por el alcohol y el 53,7\% gusto moderado. El puntaje medio acumulado en las cuatro expectativas establecidas es de 12,3 (IC95\%, 11,7 a 13,0). La edad media de inicio en el consumo de alcohol fue de 15,6 años (IC95\% $15,1$ a 16,1$)$ y la de la primera embriaguez de 16,5 años (IC95\%, 16,0 a 17,0); la diferencia media fue de un año.

Entre tipos de consumidores todas las variables son estadísticamente diferentes $(p<0,001)$, con excepción de la clasificación por el IMC y la modificación de la alimentación por recomendación médica $(p>0,05)$

\section{Expectativas positivas hacia al consumo de alcohol}

El36,3\%delossujetostieneexpectativaspositivas hacia el consumo de alcohol relacionadas con el incremento de la expresividad y la sexualidad, el $48,6 \%$, expectativas positivas relacionadas con la disminución de la tensión física, el 83,6\% con la desinhibición y los sentimientos de poder y el $56,5 \%$ con la disminución de la tensión psicológica.

Las mujeres tienen el $45,7 \%$ y los hombres $51,7 \%$ de expectativas positivas relacionadas con la disminución de la tensión física $(p<0,001)$. La anterior diferencia también se observa con las 
expectativas relacionadas con la desinhibición y los sentimientos de poder y la disminución de la tensión psicológica, alcanzándose en los hombres el $86,7 \%$ y $60,9 \% \quad(p=0,004)$, y en las mujeres, el $80,5 \%$ y $52,4 \% \quad(p=0,003)$, respectivamente. Los sujetos con 16 años o menos de edad tienen mayores puntajes en todas las expectativas $(p<0,001)$.

La cantidad de alcohol consumido semanalmente se relaciona únicamente con el puntaje relacionado con la disminución de la tensión psicológica $(p=0,024)$. En el cuadro 2 se puede apreciar la cantidad de alcohol consumido semanalmente en relación con la clasificación positiva o negativa en las expectativas y los diferentes tipos de consumo de alcohol (cuadro 3).

\section{Características del consumo problemático y del riesgo de alcoholismo según CAGE}

La proporción de consumo problemático diferente a la dependencia en la muestra fue de 13,9\%; el riesgo de los hombres es 2,9 veces (IC95\% 2,1 a 4,0) el de las mujeres. El riesgo alto de alcoholismo fue de 9,8\% para toda la población, $15,5 \%$ en los hombres y $4,3 \%$ en las mujeres (OR 3,6) (IC95\% 2,4 a 5,5).

\section{Variables asociadas al tipo de consumo}

El modelo logístico multinomial tuvo como categoría de base el tipo $A$; éste mostró cómo cuatro variables se asocian al tipo $B$ de consumo y seis al tipo C. El tipo B de consumo no está relacionado con las expectativas positivas hacia el consumo, mientras que en el tipo $\mathrm{C}$, el incremento de la expresividad y la sexualidad junto con la desinhibición y los sentimientos de poder, sí.

Los sujetos con expectativas positivas relacionadas con el consumo, la expresividad y la sexualidad tienen 1,6 veces (IC95\%; 1,0 a 2,5) más probabilidad de ser de tipo $\mathrm{C}$ que aquéllos sin ellas; en relación con la desinhibición y los sentimientos de poder positivos, este riesgo es aún mayor, de 2,2 (IC95\% 1,1 a 4,3).

La edad en una relación inversa, es factor protector para ser clasificado como consumidor de tipo $\mathrm{B}$ o $\mathrm{C}$. Los hombres tienen mayor riesgo que las mujeres de ser clasificados como de tipo $\mathrm{B}$ ○ $\mathrm{C}$, al igual que los sujetos que manifiestan gusto moderado o elevado por las bebidas alcohólicas.

Cuadro 2. Cantidad de alcohol consumido semanalmente $(g)^{\star}$ por tipo de consumo de alcohol, según las expectativas hacia el consumo.

\begin{tabular}{|c|c|c|c|}
\hline \multirow[b]{2}{*}{ Expectativa } & \multicolumn{3}{|c|}{ Tipo de consumo † I } \\
\hline & $\begin{array}{c}\text { Tipo A } \\
\text { [475] }\end{array}$ & $\begin{array}{c}\text { Tipo B } \\
\text { [218] }\end{array}$ & $\begin{array}{c}\text { Tipo C } \\
{[506] \neq}\end{array}$ \\
\hline \multicolumn{4}{|c|}{ Expresividad y sexualidad } \\
\hline Negativa [764] & $13,4(12,5 \text { a } 14,3)^{*}$ & $54,0(49,4$ a 59,0$)$ & $89,7(74,0$ a 108,6$)$ \\
\hline Positiva [435] & $13,7(12,3$ a 15,3$)$ & $47,9$ (42,2 a 54,2$)$ & $78,2(64,2$ a 95,2$)$ \\
\hline \multicolumn{4}{|c|}{ Disminución tensión física } \\
\hline Negativa [616] & $13,5(12,5$ a 14,5$)$ & $52,2(47,3$ a 57,6$)$ & $84,3(67,8$ a 104,7$)$ \\
\hline Positiva [583] & $13,6(12,4$ a 14,8$)$ & $51,8(46,6$ a 57,7$)$ & $83,2(69,6$ a 99,3$)$ \\
\hline \multicolumn{4}{|c|}{ Desinhibición y poder } \\
\hline Negativa [197] & $13,9(12,0$ a 16,0$)$ & $49,0(41,2$ a 58,3$)$ & $87,4(41,7$ a 184,4$)$ \\
\hline Positiva [1 002] & $13,4(12,6$ a 14,3$)$ & $52,7(48,6$ a 57,0$)$ & $83,3(72,5$ a 95,8$)$ \\
\hline \multicolumn{4}{|c|}{ Disminución tensión psicológica } \\
\hline Negativa [521] & $13,6(12,6$ a 14,7$)$ & $51,2(45,9$ a 57,0$)$ & $80,8(63,6$ a 102,7$)$ \\
\hline Positiva [678] & $13,4(12,3$ a 14,6$)$ & $52,7(47,8$ a 58,2$)$ & $84,9(71,8$ a 100,4$)$ \\
\hline
\end{tabular}

\footnotetext{
* Promedio e intervalo de confianza del 95\%. Con base en el logaritmo de los gramos/semana, consumidos de alcohol. $†$ Tipo de consumo: A, no excede el límite de unidades internacionales de consumo semanal de alcohol y es negativo para CPA y CAGE; B, excede el límite de unidades internacionales de consumo semanal de alcohol y es negativo para CPA y CAGE; C: es positivo para CPA, CAGE o ambos, sin importar si excede o no el límite de unidades internacionales de consumo semanal de alcohol. $¥$ [n]. | Según ANOVA (cantidad-tipo-expectativa); $\mathrm{F}<0,001$ para todos los tipos; F $>0,05$ para todas las expectativas.
} 
Cuadro 3. Puntaje alcanzado según el sexo y el tipo de consumo de alcohol en las expectativas positivas hacia el consumo de alcohol, ajustado por edad, escolaridad y nivel socioeconómico.

\begin{tabular}{|c|c|c|c|c|c|c|}
\hline $\begin{array}{l}\text { Tipo de consumo * } \\
\text { Expectativa }\end{array}$ & Tipo A & $\begin{array}{c}\text { Hombre } \\
\text { Tipo B }\end{array}$ & Tipo C & Tipo A & $\begin{array}{l}\text { Mujer } \\
\text { Tipo B }\end{array}$ & Tipo C \\
\hline \multicolumn{7}{|c|}{ Incremento de la expresividad y la sexualidad (rango $0-4$ ) } \\
\hline Puntaje | & $1,9(1,7$ a 2,1$) \dagger$ & $1,9(1,8$ a 2,1$)$ & $2,4(2,2$ a 2,6$)$ & $1,8(1,7$ a 1,9$)$ & $1,9(1,7$ a 2,1$)$ & $2,4(2,1$ a 2,7$)$ \\
\hline Negativa $(<2 p$ & $103\{27,9\} \ddagger$ & $187\{50,5\}$ & $80\{21,6\}$ & $226\{57,4\}$ & $141\{35,8\}$ & $27\{6,8\}$ \\
\hline Positiva | & $54\{24,8\}$ & $83\{38,1\}$ & $81\{37,1\}$ & $123\{56,7\}$ & $64\{29,5\}$ & $30\{13,8\}$ \\
\hline \multicolumn{7}{|c|}{ Disminución de la tensión física (rango 0-5) } \\
\hline Puntaje & $1,4(1,2$ a 1,7$)$ & $1,8(1,6$ a 2,0$)$ & $2,0(1,8$ a 2,3$)$ & $1,6(1,4$ a 1,7$)$ & $1,6(1,4$ a 1,8$)$ & $1,8(1,4$ a 2,2$)$ \\
\hline Negativa (<1 puntos) & $86\{30,3\}$ & & $65\{22,9\} \mid$ & $193\{58,1\}$ & $34,0\}$ & $26\{7,8\}$ \\
\hline Positiva & $71\{23,4\}$ & $137\{45,0\}$ & $96\{31,6\} \mid$ & $156\{55,9\}$ & $92\{33,0\}$ & $31\{11,1\}$ \\
\hline \multicolumn{7}{|c|}{ Desinhibición y sentimientos de poder (rango; 0-9) } \\
\hline Puntaje & $4,6(4,3$ a 4,9$)$ & $4,7(4,5$ a 5,0$)$ & $5,5(5,2$ a 5,9 & ) $4,5(4,2$ a 4,7$)$ & $4,7(4,4$ a 5,1$)$ & $5,7(5,1$ a 6,3$)$ \\
\hline Negativa (<2 puntos) & $86\{30,3\}$ & $133\{46,8\}$ & $65\{22,9\} \mid$ & $193\{58,1\}$ & $113\{34,0\}$ & $26\{7,8\}$ \\
\hline Positiva & $71\{23,4\}$ & $137\{45,0\}$ & $96\{31,6\}$ & $156\{55,9\}$ & $92\{33,0\}$ & $31\{11,1\}$ \\
\hline \multicolumn{7}{|c|}{ Disminución de la tensión psicológica (rango 0-4) } \\
\hline Puntaje & $1,7(1,5$ a 1,9$)$ & $1,9(1,8$ a 2,1$)$ & $2,4(2,1$ a 2,6$)$ & $1,6(1,5$ a 1,7$)$ & $1,8(1,6$ a 2,0$)$ & $2,3(1,9$ a 2,6$)$ \\
\hline Negativa (<1 puntos) & $66\{28,7\}$ & $117\{50,9\}$ & $47\{20,4\}$ & $172\{59,1\}$ & $101\{34,7\}$ & $18\{6,2\}$ \\
\hline Positiva| & $91\{25,4\}$ & $153\{42,8\}$ & $114\{31,8\}$ & $177\{55,3\}$ & $104\{32,5\}$ & $39\{12,2\}$ \\
\hline
\end{tabular}

* Tipo de consumo: A, no excede el límite de unidades internacionales de consumo semanal de alcohol y es negativo para CPA y CAGE; B, excede el límite de unidades internacionales de consumo semanal de alcohol y es negativo para CPA y CAGE; C, es positivo para CPA, y/o CAGE o ambos, sin importar si excede o no el límite de unidades internacionales de consumo semanal de alcohol. † Promedio e intervalo de confianza del 95\%. ‡ Número $\{\%\}$. | $p<0,05$.

El nivel socioeconómico presenta un comportamiento disímil: mientras el nivel más alto de la escala socioeconómica es un factor de riesgo para presentar el tipo $\mathrm{B}$, los niveles medio y alto son factores protectores para ser clasificado como de tipo C. El modelo explicativo presentado fue congruente cuando se aplicó a los menores de 24 años, los sujetos entre 25 y 40 años y los mayores de 40 años.

El cuadro 4 presenta las OR y los intervalos de confianza para estas variables, como también los índices de bondad de ajuste que evidencian la calidad de los modelos propuestos.

\section{Discusión}

\section{Alcance y limitaciones}

Este estudio es el resultado de diez años de investigación aplicada en el tema; en él se incorporaron el conocimiento y la experiencia previamente adquiridos, y la tecnología diagnóstica desarrollada y validada en esta población $(8-11,14,24)$. Las limitaciones del estudio son las propias de la investigación epidemiológica en poblaciones. Sin embargo, todos los posibles sesgos fueron advertidos y controlados, y se hizo especial énfasis en garantizar un tamaño de muestra adecuado y en la calidad de las mediciones. Por lo anterior, los resultados son la mejor expresión hasta ahora lograda del consumo de alcohol y sus variables asociadas en la población de Bucaramanga.

\section{La importancia de una clasificación del consumo}

Los resultados presentados cobran relevancia en la medida en que son producto de un proceso en el cual la correcta medición fue garantizada previamente $(9,11)$. Se discriminaron tres tipos de consumidores de alcohol, con suficiente poder discriminatorio para ser utilizados en estudios epidemiológicos. La estrategia utilizada permitió, de un lado, integrar aspectos que a priori pueden considerarse como separados: la cantidad de alcohol, el consumo problemático y la dependencia, pero que, en la dinámica del 
Cuadro 4. Razón de disparidad para la prevalencia (OR), obtenida en un modelo logístico multinomial para explicar el tipo de consumo de alcohol.

\begin{tabular}{|c|c|c|}
\hline & OR (IC 95\%) † & Valor $p$ \\
\hline \multicolumn{3}{|l|}{ Tipo B } \\
\hline Incremento de la expresividad y sexualidad (positiva) & $0,88(0,62$ a 1,25$)$ & 0,494 \\
\hline Disminución tensión física (positiva) & $1,21(0,88$ a 1,67$)$ & 0,242 \\
\hline Desinhibición y sentimientos de poder (positiva) & $1,11(0,74$ a 1,66$)$ & 0,613 \\
\hline Disminución de la tensión psicológica (positiva) & $0,92(0,64$ a 1,32$)$ & 0,651 \\
\hline Edad (por cada cinco años) & $0,85(0,80$ a 0,90$)$ & 0,000 \\
\hline \multicolumn{3}{|l|}{ Sexo } \\
\hline Mujer & 1,0 & \\
\hline Hombre & $2,47(1,85$ a 3,29$)$ & 0,000 \\
\hline Nivel socioeconómico & $\ddagger$ & \\
\hline Uno & 1,0 & \\
\hline Dos & $1,30(0,92$ a 1,84$)$ & 0,136 \\
\hline Tres & $1,64(1,14$ a 2,35$)$ & 0,007 \\
\hline \multicolumn{3}{|l|}{ Gusto manifestado por el trago } \\
\hline Poco & 1,0 & \\
\hline Moderado & $4,21(3,04$ a 5,84$)$ & 0,000 \\
\hline Mucho & $4,15(1,70$ a 10,18$)$ & 0,003 \\
\hline \multicolumn{3}{|c|}{ Hosmer-Lemeshow $\left({ }^{\wedge} C\right) ; 5,21(p=0,734)$ : Pearson $(X 2) ; 975,4(p=0,401)$} \\
\hline \multicolumn{3}{|l|}{ Tipo C } \\
\hline Expresividad y sexualidad (positiva) & $1,57(1,00$ a 2,46$)$ & 0,049 \\
\hline Disminución tensión física (positiva) & $1,26(0,82$ a 1,93$)$ & 0,290 \\
\hline Desinhibición y sentimientos de poder (positiva) & $2,17(1,09$ a 4,32$)$ & 0,027 \\
\hline Disminución de la tensión psicológica (positiva) & $0,94(0,58$ a 1,54$)$ & 0,816 \\
\hline Edad (por cada cinco años) & $0,73(0,67$ a 0,79$)$ & 0,000 \\
\hline \multicolumn{3}{|l|}{ Sexo } \\
\hline Mujer & 1,0 & \\
\hline Hombre & $4,70(3,13$ a 7,04$)$ & 0,000 \\
\hline \multicolumn{3}{|l|}{ Nivel socioeconómico } \\
\hline Uno & 1,0 & \\
\hline Dos & $0,70(0,44$ a 1,12$)$ & 0,138 \\
\hline Tres & $0,79(0,48$ a 1,28$)$ & 0,339 \\
\hline \multicolumn{3}{|l|}{ Gusto manifestado por el trago } \\
\hline Poco & 1,0 & \\
\hline Moderado & $7,73(5,01$ a 11,93$)$ & 0,000 \\
\hline Mucho & $23,54(9,49$ a 58,46$)$ & 0,000 \\
\hline Hosmer-Lemeshow $\left({ }^{\wedge} \mathrm{C}\right) ; 1,5(p=0,992)$ : Pearson $(X$ & & \\
\hline
\end{tabular}

*. Tipo de consumo: A, no excede el límite de unidades internacionales de consumo semanal de alcohol y es negativo para CPA y CAGE; B, excede el límite de unidades internacionales de consumo semanal de alcohol y es negativo para CPA y CAGE; C, es positivo para CPA y/o CAGE, sin importar si excede o no el límite de unidades internacionales de consumo semanal de alcohol. † Razón de prevalencia e intervalo de confianza del 95\%. ‡ Con base en la oficina de planeación municipal, el nivel bajo corresponde a los estratos socioeconómicos 1 y 2 , el medio a los estratos 3 y 4 , y el alto a los estratos 5 y 6 .

consumidor son inseparables, y de otro, añadir un perfil biológico, sociodemográfico y dietético a las mismas.

El desarrollo de los tipos ha sido sustentado en diferentes dimensiones; la personalidad de los consumidores ha sido una de ellas. Actualmente, siguen vigentes dos propuestas de esta perspectiva: la planteada por Goldberg y basada en el léxico, y la planteada por Costa y McCrae y basada en la tradición de los cuestionarios $(34,35)$. Sin embargo, con ninguna de ellas se han podido establecer relaciones causales sólidas; incluso, se ha sugerido que la personalidad es consecuencia del consumo de alcohol (35).

Se han desarrollado otras clasificaciones para los sujetos alcohólicos y, de esta forma, pronosticar y evaluar su tratamiento. El método propuesto 
por Cloninger para clasificar los alcohólicos en tipo I, II y otros subtipos, ha permitido conocer en detalle el camino recorrido por los sujetos hasta convertirse en alcohólicos y la posibilidad de recidiva (36). La determinación de estilos de consumo, entre jóvenes y adultos, es un paso intermedio para establecer categorías como "consumo normal" o "problemático" que, si bien discriminan, no han permito tampoco establecer relaciones de tipo causal (37).

Otras aproximaciones desde la perspectiva cultural proponen sociedades que pueden identificarse como abstemias, bebedoras en rituales, bebedoras triviales, banales o que persiguen la embriaguez. Estas clasificaciones, a pesar de permitir la identificación de grupos sociales y el estudio de las relaciones morales y normativas con el alcohol (5), no han sido exploradas en relaciones causales.

Desde la perspectiva epidemiológica, se han intentado clasificaciones relacionadas con la obesidad, la hipertensión, los eventos cardiovasculares, el cáncer, los accidentes en vehículo automotor y otros problemas considerados de salud pública $(1,18,38)$. De esta perspectiva han surgido categorías para clasificar sujetos con base en la historia del consumo, el número de tragos, la frecuencia de consumo o el comportamiento parrandero (binge) y el tipo de bebida consumida. Sin embargo, estas clasificaciones tienen poco poder discriminatorio, no incorporan la dinámica del consumo de alcohol $y$, en la práctica epidemiológica, presentan dificultad por la gran cantidad de error que incorporan.

\section{Variables asociadas al tipo de consumo}

Previamente se estableció las variables para esta población que se asocian con el consumo de alcohol. Se concluyó que está íntimamente relacionado con el contexto social y el comportamiento grupal, más que con el individual (14).

Sin embargo, a la luz de estos resultados y del avance al establecer clasificaciones que conceptualmente son sólidas e incorporan todo el espectro de la dinámica del consumo y sus consecuencias, podría afirmarse que la explicación previa fue limitada. Mientras ahora seis variables explican al tipo de consumo, sólo dos explicaron el hecho de consumir alcohol (14). Se ratificó que en esta población las expectativas positivas hacia el consumo son las principal explicación de las consecuencias indeseables al consumir alcohol; ahora es claro que la relación del alcohol con expectativas positivas hacia la expresividad verbal y la sexualidad, y la desinhibición y los sentimientos de poder, son factores de riesgo para el consumo problemático y la dependencia.

La edad de inicio y la de la primera embriaguez son consecuencia de estas expectativas 0 mediadoras que incrementan el puntaje en las mismas; la edad en relación inversa con la probabilidad de presentar tipos no deseables de consumo, es la expresión matemática de lo anterior. La edad de inicio ha sido un importante factor de predicción del consumo y de las consecuencias negativas (13,39); aquí se estableció que el tiempo que transcurre entre el inicio y la primera embriaguez también lo es.

El sexo está asociado con el hecho de presentar exceso de consumo y, posteriormente, consumo problemático; sin embargo, la experiencia previa mostró que no lo es para consumir alcohol; esto es coherente con lo ya conocido en el sentido de que en la preadolescencia se ratifica el rol de género y se diferencia el patrón de comportamiento en la sociedad (13,40-42).

Igual sucede con el gusto manifestado por alcohol; éste tiene dos componentes: el simbólico hacia la bebida, que le confiere estatus, íntimamente relacionado con el poder y previo al primer consumo, y el ratificado con el consumo. El gusto por el alcohol es un aprendizaje que se facilita por puntajes altos en las expectativas positivas, el reforzamiento social del consumo y la edad temprana de inicio, pues en los jóvenes los mecanismos de autodeterminación y valoración del proyecto de vida aún no están consolidados.

La forma como influye el nivel socioeconómico aún no es clara: en el tipo $B$, que presenta consumo excesivo y es previo al consumo 
problemático y la dependencia, podría operar por la vía de una mayor capacidad de acceso a las bebidas por mayor ingreso; en el tipo C podría operar como protector, representando a otras variables no estudiadas y relacionadas con el bienestar económico, la satisfacción personal y la articulación social.

\section{Implicación de los resultados}

Ahora se cuenta con herramientas de medición $(9-11,24)$, y explicaciones matemáticas y conceptuales que permiten explicar cómo se establecen las relaciones entre el alcohol y los sujetos en esta población, y sobre cómo puede, dentro de esa dinámica, llegarse al consumo excesivo de alcohol, el consumo problemático y la dependencia.

La teoría de las expectativas es un concepto sólido que, por la manera como se generan y operan éstas, tiene la capacidad de predecir a manera de explicación causal $(13,14,24)$. Los resultados aquí presentados y los otros hasta ahora logrados, deben traducirse en intervenciones en la población y en grupos de riesgo como los escolares y preadolescentes, con enfoque de género.

Talvezelprincipalelementoquesedebeconsolidar en los sujetos objeto de la intervención es el de la autoestima, pues este concepto también se construye como el de las expectativas, es decir, desde una perspectiva cognitivo conductual y dentro del aprendizaje social y cultural $(41,43)$. Las metas centrales de dicha intervención deben ser aumentar la valoración positiva de sí y la capacidad de autodeterminarse, lo que, en teoría, traduciría puntuaciones bajas en las expectativas asociadas.

El impacto buscado sería la disminución en la edad de inicio en el consumo de alcohol, la pérdida de estatus simbólico del hecho de beber y de las bebidas alcohólicas, y el espaciamiento entre el inicio en el consumo y la primera embriaguez.

\section{Conflicto de interés}

Los autores declaran que no tienen conflicto de interés de ningún tipo, ni real o potencial sobre los resultados presentados.

\section{Financiación}

El presente trabajo fue cofinanciado por la Universidad Industrial de Santander (código interno UIS 8656) y el Instituto Colombiano para el Desarrollo de la Ciencia y la Tecnología "Francisco José de Caldas", Colciencias. El proyecto fue seleccionado mediante convocatoria pública y asignado el código 1102-345-18973.

\section{Referencias}

1. Miller TR, Levy DT, Spicer RS, Taylor DM. Societal costs of underage drinking. J Stud Alcohol. 2006;67: 519-28.

2. Konnopka A, König HH. Direct and indirect costs attributable to alcohol consumption in Germany. Pharmacoeconomics. 2007;25:605-18.

3. Stranges S, Wu T, Dorn JM, Freudenheim JL, Muti P, Farinaro E, et al. Relationship of alcohol drinking pattern to risk of hypertension a population-based study. Hypertension. 2004;44:813-9.

4. Uekermann J, Daum I. Social cognition in alcoholism: a link to prefrontal cortex dysfunction? Addiction. 2008;103:726-35.

5. Room R, Mäkelä K. Typologies of the cultural position of drinking. J Stud Alcohol. 2000;61:475-83.

6. Lesch OM, Kefer J, Lentner S, Mader R, Marx B, Musalek M, et al. Diagnosis of chronic alcoholismclassificatory problems. Psychopathology. 1990;23:8896.

7. Dobson R.Governmentmustgettoughonalcoholmisuse, public health experts warn. BMJ. 2008;336:1266-7.

8. Herrán OF, Ardila MF. Consumo de alcohol, riesgo de alcoholismo y alcoholismo en Bucaramanga, Colombia, 2002-2003. Colombia Med. 2005;36:158-67.

9. Herrán OF, Ardila MF. Validity and reproducibility of two semi-quantitative alcohol frequency questionnaires for Colombian population. Public Health Nutr. 2006;9:76370.

10. Herrán OF, Ardila MF. Validez interna y reproducibilidad de la prueba CAGE en Bucaramanga, Colombia. Biomédica. 2005;25:231-41.

11. Herrán OF, Ardila MF, Barba DM. Consumo problemático de alcohol en Bucaramanga, Colombia: diseño y validación de una prueba. Biomédica. 2008;28:25-37.

12. Brown SA, Goldman MS, Inn A, Anderson LR. Expections of reinforcement from alcohol their domain and relation to drinking patterns. J Consult Clin Psychol. 1980;48:419-26.

13. Goldman MS, Del Boca FK, Darles J. Alcohol expectancy theory: The application of cognitive neuroscience. En: Leonard KE, Blane HT, editors. Psychological theories 
of drinking and alcoholism. Second edition. New York: Guilford Press; 1999. p. 203-46.

14. Ardila MF, Herrán OF. Expectativas en el consumo de alcohol en Bucaramanga, Colombia. Rev Med Chil. 2008;136:73-82.

15. Penick EC, Powell BJ, Nickel EJ, Read MR, Gabrielli WF, Liskow BI. Examination of Cloninger's type I and type II alcoholism with a sample of men alcoholics in treatment. Alcohol Clin Exp Res. 1990;14:623-9.

16. Farren CK, Dinan TG. Alcoholism and typology: findings in an Irish private hospital population. J Stud Alcohol. 1996;57:249-52.

17. Colder CR, Chassin L. The psychosocial characteristics of alcohol users versus problem users: data from a study of adolescents at risk. Dev Psychopathol. 1999;11:32148.

18. Arif AA, Rohrer JE. Patterns of alcohol drinking and its association with obesity: data from the Third National Health and Nutrition Examination Survey, 1988-1994. BMC Public Health. 2005;5:126.

19. Rouillier P, Boutron-Ruault MC, Bertrais S, Arnault N, Daudin JJ, Bacro JN, et al. Drinking patterns in French adult men-a cluster analysis of alcoholic beverages and relationship with lifestyle. Eur J Nutr. 2004;43:69-76.

20. Carrasco AM. Consumo de alcohol y estilos de vida: una tipología de los adolescentes españoles. Revista de Psicología Social. 2004;19:51-79.

21. Colder CR, Chassin L. The psychosocial characteristics of alcohol users versus problem users: data from a study of adolescents at risk. Dev Psychopathol. 1999;11:32148.

22. Norman G, Streiner D. Bioestadística. Barcelona: Editorial Mosby; 1996. p. 129-48.

23. Tavella NM. Análisis de los ítems en la construcción de instrumentos psicométricos. México D.F.: Editorial Trillas; 1978. p. 75.

24. Herrán OF, Ardila MF. Validez factorial del cuestionario de expectativas hacia el consumo de alcohol (AEQ-III), en adultos de Bucaramanga, Colombia. Rev Med Chil. 2007;135:488-95.

25. Wall AM, Thrussell C, Lalonde RN. Do alcohol expectancies become intoxicated outcomes? A test of social-learning theory in a naturalistic bar setting. Addictive Behav. 2003;28:1271-83

26. González J. Alcohol. Tipos de intervención en el consumo de riesgo. Fecha de consulta: 20 de agosto de 2008. Disponible en:http://www.seslap.com/seslap/html/ pubBiblio/revista/n_8/n8Art2.pdf

27. Greenland S. Modeling and variable selection in epidemiologic analysis. Am $J$ Public Health. 1989;79:340-9.
28. Hosmer DW, Lemeshow S. Applied logistic regression. Second edition. New York: John Wiley \& Sons, Inc.; 2000.

29. Long JS. Regression models for categorical and limited dependent variables. London: SAGE Publications; 1997. p. $148-86$.

30. Centro para el Control de las Enfermedades. Epilnfo, versión 6.04d. Epidemiología en ordenadores. Atlanta, Georgia: Centro para el Control de las Enfermedades; 2001.

31. Eltinge JL, Sribney WM. Estimation of means, totals, ratios, and proportions for survey data. Stata Technical Bulletins. 1996;6:213-35.

32. Levy SP, Lemeshow S. Sampling of populations: Methods and applications. Third edition. New York: Jhon Wiley \& Sons; 1999. p. 365-90.

33. StataCorp. Stata Statistical Software: Release 10.1 Collegue Station, TX: StataCorp LP; 2008.

34. Goldberg LR. The development of markers for the Bigfive factor structure. Psychol Assess. 1992:4;26-42.

35. Sher KJ, Trull TJ, Bartholow BD, Vieth A. Personality and alcoholism: issues, methods, and etiological processes. En: Leonard KE, Blane HT, editors. Psychological theories of drinking and alcoholism. Second edition. New York: Guilford Press; 1999. p. 54-105.

36. Penick EC, Powell BJ, Nickel EJ, Read MR, Gabrielli WF, Liskow BI. Examination of Cloninger's type I and type II alcoholism with a sample of men alcoholics in treatment. Alcohol Clin Exp Res. 1990;14:623-9.

37. Kilty KM. Drinking styles of adolescents and young adults. J Stud Alcohol. 1990;51:556-64.

38. Fan AZ, Russell M, Stranges S, Dorn J, Trevisan M. Association of lifetime alcohol drinking trajectories with cardiometabolic risk. J Clin Endocrinol Metab. 2008;93:154-61.

39. Pelucchi C, Gallus S, Garavello W, Bosetti C, La Vecchia C. Alcohol and tobacco use, and cancer risk for upper aerodigestive tract and liver. Eur J Cancer Prev. 2008;17:340-4.

40. Mora J, Natera G. Expectativas, consumo de alcohol y problemas asociados en estudiantes universitarios de la ciudad de México. Salud Pública Mex. 2001;43:89-96.

41. Wild LG, Flisher AJ, Bhana A, Lombard C. Associations among adolescent risk behaviours and self-esteem in six domains. J Child Psychol Psychiatry. 2004;45:1454-67.

42. Medina-Mora ME, Borges G, Villatoro J. The measurement of drinking patterns and consequences in Mexico. J Subst Abuse. 2000;12:183-96.

43. Wild LG, Flisher AJ, Bhana A, Lombard C. Substance abuse, suicidality, and self-esteem in South African adolescents. J Drug Educ. 2004;34:1-17. 